\title{
Research on Cloud Safe Storage Evaluation Model from Perspective of Internet Finance
}

\author{
$\mathrm{Hu}$ yanqiang \\ Fudan University of management, Postdoctoral research center of Management \\ Science and Engineering \\ huyanqiang@126.com
}

\begin{abstract}
Cloud storage plays a role of file management and store backups with characteristics of scalability and flexibility. With increment of the formation the clients store in the cloud, cloud safe storage from perspective of internet finance becomes the hot spot in the current researches. This paper proposes the cloud safe storage evaluation model, builds the fuzzy math comprehensive evaluation system from perspective of Internet finance, and establishes evaluation matrix in order to calculate discrete assessment values based on expert evaluation mechanism. The product of the weight vector and the evaluation vector is considered the comprehensive evaluation results of cloud storage model finally by the evaluation model. Secondly, taking a comparable analysis on HDFS safety storage model, DES encryption cloud safety storage model and AES encryption cloud safety storage model that are based on encrypting, will then provide a theoretical direction for customers to select suitable storage service.
\end{abstract}

Keywords: Internet finance, Cloud security, Evaluation model, HDFS

\section{Introduction}

With widespread use of cloud computing, data security in a cloud environment is paid more and more attention by the public gradually. Required by large amounts of information, data privacy and security play an increasing significant role in people's daily life as the consequences of important information's being stolen by terrorist would be horrific. ${ }^{[1]}$

There are great differences in measures between traditional data protection and the current cloud security protection. Generally the traditional data protection mainly focuses on hardware, but it is far from sufficient to just break the integrity of data as there is high safety risk of distributed applications between virtualized servers in the cloud security mode. ${ }^{[2]}$ There once was system breakdown of six hours in Amazon of 2008, making the system unavailable to its users mainly as damages in service component of the computer lead to failure in running storage services; Gmail' server also once failed in running in 2009, making the engine unavailable to the users; in the year of 2010, there was a phenomenon of downtime in the data back-up center, making a loss of some data. Such internet security incidents reduce the users' trust to great extent. ${ }^{[3]}$ Therefore, the relevant experts built the Cloud Security Alliance as well as the cloud safety risk model; some companies such as Intel give technical specification and practical significance aimed at the architecture frame in the environment of cloud security; Santos and others set up runtime environment for the users aimed at TCCP, a cloud computing platform and design a virtual machine there into for private information protection. ${ }^{[4]}$

In view of proposal of various cloud safe storage evaluation models recently, this paper researches the cloud safe storage evaluation model under the environment of Internet based on the previous researches, computes the results of the evaluation model 
with the quantification method, compares uniformly and evaluates the performance between the current various models. ${ }^{[5]}$

\section{Analysis of Internet finance's characteristics}

With development of social economy, the Internet has been essential in people's daily life. It's worth mentioning that Internet finance affects people in their daily life in its spirit of 'Openness, equality, collaboration and sharing. The current Internet finance is a new industry combining traditional finance and the spirit of Internet. The application of computer networks IN developing finance also has the following characteristics in the Internet finance mode:

Table 1. Characteristics of Internet Finance

\begin{tabular}{cc}
\hline Characteristics & Detailed Description \\
\hline $\begin{array}{c}\text { Rapid } \\
\text { Development }\end{array}$ & The scale develops quickly based on big data and e-business \\
High Efficiency & The transaction process is simple, quick and standard by processing data with \\
computers & \\
Low Cost & The median and transaction cost are unnecessary in the transaction through \\
online platforms without monopoly profits. \\
Big Risk \\
$\begin{array}{c}\text { Weak } \\
\text { Management }\end{array}$
\end{tabular}

\section{Research on the Cloud Safe Storage Evaluation Model}

\subsection{Analysis of the Model Principle}

There exist many influential factors on cloud storage. According to the faction index, it could be classified into quantifiable one and unquantifiable one. Those quantitative factors, such as dimension and time factor, could be calculated quantitatively, while most of the factors can not. In blurry comprehensive evaluation, evaluation objects and function index related to evaluation factors as well as the evaluation rank should be assured firstly. Secondly, the weight and subordination vector of each influential factor should be assured secondly, then we will get blurry evaluation array. Evaluation results will be quirked by calculating weight vectors and blurry evaluation array.

\subsection{Model Building and Soluting}

(1) Assure the evaluation muster

Blurry layer analysis is using math that study and deal with blurry phenomenon through mathematical methods. Nowadays the application of blurry layer analysis has been popular in all fields of theory, mechanisms, farming, medical and social sciences, which fully indicates its strong vitality and permeating force. The principle of the model is using linear converting and combining with the biggest subordination degree and doing comprehensive consideration and research with taking many factors into consideration to arrive a rather suitable evaluation results. Therefore, the methods and steps of social evaluation methods is displayed as the following:

Firstly, the evaluation object should be assured, its variable is influenced by y divisors, and its divisor array is " $\mathrm{V}$ ", we define it as :

$$
V=\left(V_{1,} V_{i 2}, V_{3}, \cdots, V_{\mathrm{n}}\right)
$$




$$
\text { And we rule: } \quad V_{i}(i=1,2,3, \cdots, y)
$$

On account of the difference of each evaluation index, some of the evaluations fail to have quantitative analysis, thus adopting statistics evaluation measures. Evaluation methods are generally evaluated by experts according to country standard evaluation mechanism. the evaluation array is:

$$
U=\{A, B, C, D, E\}
$$

(2) Weight ratio and subordination function

On account of the different weight of each variable, so the influential extent of assured evaluation degree is varied, we assume the weight allocation as $\mathrm{P} 1$, and

$$
\begin{array}{rr} 
& P_{i}=\left(P_{1} P_{2}, P_{3}, \cdots P_{y}\right) \\
\text { In it } & P_{i}(i=1,2,3, \cdots, y)
\end{array}
$$

is the weight result of (2),based on the common sense we know w1>=o, and

$$
\sum_{i=1}^{y} W_{i}=1
$$

In order to calculate the evaluation array, subordinating function is led in to integrate disperse evaluation results into a continuous one and fetch $[0,1]$, when the subordination results $\mathrm{A}(\mathrm{x})$ in subordination function is lower with the change of value, the extent of $\mathrm{X}$ belongs to $\mathrm{A}$ is then proved to be lower. This essay has adopted logarithmic function and cauthy distribution function as subordination function, the functional relation is:

$f(x)=\left\{\begin{array}{ll}{\left[1+\alpha(x-\beta)^{-2}\right]^{-1},} & 1 \leq x \leq 3 \\ \alpha \ln x+b, & 3 \leq x \leq 5\end{array} \quad \alpha, \quad \beta, a, \quad b\right.$ are all constants.

(3) The calculation of evaluation results

According to the above relation and the experts ' statically results, we get generating evaluation spastically array. Comprehensive results and weight can get first-grade performance evaluation value. Making $\mathrm{D}=\mathrm{f}(\mathrm{Xi})$, we can get the model evaluation results using weight vector minus evaluation vector

\section{Score $=D \bullet P^{T}$}

Given there are five grades in evaluating one factor by various people, $\left\{v_{1}, v_{2}, v_{3}, v_{4}, v_{5}\right\}$ and each grade is set respectively 5,4,3,2, 1. Define the constants in term of membership function, ${ }^{[4]}$ In condition of $A(x=5)$, the membership is $1, f(5)=1$;

In condition of $C(x=3)$, the membership is $0.8, f(3)=0.8$;

In condition of $D(x=1)$, the membership is $0.01, f(0)=0.01$;

The constants obtained are respectively: $\alpha=1.1086, \beta=0.8942, a=0.3915$, $b=0.3699$ and then a membership function can be obtained:

$$
f(x)=\left\{\begin{array}{l}
{\left[1+1.1086(x-0.8924)^{-2}\right]^{-1}, 1 \leq x \leq 3} \\
0.3915 \ln x+0.3699,3 \leq x \leq 5
\end{array}\right.
$$

\section{Cloud Safe Storage Evaluation}

Based on cloud safety storage evaluation model, this experiment sets the evaluation of HDFS cloud safety storage as an example 


\subsection{The research on HDFS}

Via the questionnaire of thirty researchers on cloud safety storage, and setting the cloud safety storage model 's economics, convenience and safety based on crypt as the evaluation index, we can get the facility influence factor results form of the model. It is indicated as the following chart:

Table 2. The Test on Economic of HDFS Cloud Safety Storage Model

\begin{tabular}{cccc}
\hline Classification & Technology investment & Storage resources & Calculating resources \\
\hline (1) & 0 & 2 & 0 \\
$(2)$ & 2 & 4 & 2 \\
$(3$ & 6 & 5 & 4 \\
$(4)$ & 7 & 5 & 4 \\
5 & 5 & 4 & 10 \\
\hline
\end{tabular}

Table 3. The Convenience Test on HDFS Cloud Safety Storage Model

\begin{tabular}{cccc}
\hline Classification & Share mechanisms & Updating mechanisms & Searching machines \\
\hline 1 & 4 & 6 & 5 \\
2 & 4 & 5 & 4 \\
$(3$ & 3 & 5 & 4 \\
4 & 6 & 2 & 5 \\
5 & 3 & 2 & 2 \\
\hline
\end{tabular}

Table 4. The Safety Test on HDFS Safety Storage Model

\begin{tabular}{cccc}
\hline Classification & Date controlled & Digital signature & Transportation rows \\
\hline 1 & 6 & 6 & 4 \\
$(2)$ & 5 & 4 & 5 \\
$(3$ & 3 & 4 & 7 \\
$(4)$ & 4 & 3 & 3 \\
5 & 2 & 3 & 1 \\
\hline
\end{tabular}

\subsection{HDFS evaluation matrix establishing and soluting}

(1)Establishment of matrix

The relevant Economy $E_{3 \times 5}$, Convenience $C_{5 \times 5}$ and Security $S_{5 \times 5}$ evaluation matrix are obtained based on the above statistical tables:

$$
D_{5 \times 5}=\left[\begin{array}{lllll}
0.05 & 0.1 & 0.2 & 0.3 & 0.2 \\
0.14 & 0.25 & 0.3 & 0.15 & 0.25 \\
0.2 & 0.25 & 0.35 & 0.15 & 0.05 \\
0.15 & 0.2 & 0.2 & 0.15 & 0.3 \\
0.3 & 0.2 & 0.15 & 0.15 & 0.1
\end{array}\right]
$$


(2)the soluting of array

$$
\begin{aligned}
C_{5 \times 5} & =\left[\begin{array}{lllll}
0 & 0.2 & 0.25 & 0.3 & 0.2 \\
0.2 & 0.2 & 0.15 & 0.25 & 0.2 \\
0.15 & 0.2 & 0.2 & 0.25 & 0.15 \\
0.3 & 0.25 & 0.25 & 0.1 & 0.1 \\
0.345 & 0.2 & 0.1 & 0.3 & 0.15
\end{array}\right] \\
E_{3 \times 5} & =\left[\begin{array}{lllll}
0 & 0.1 & 0.2 & 0.2 & 0.5 \\
0.1 & 0.2 & 0.25 & 0.25 & 0.35 \\
0 & 0.1 & 0.3 & 0.2 & 0.25
\end{array}\right]
\end{aligned}
$$

Give point values to the elements within the evaluation grade and then a evaluation vector is obtained: $Q=(5,4,3,2,1)$. The evaluation value of various performance influencing factors is the product of evaluation vector and evaluation matrix:

$$
\begin{aligned}
D_{5 \times 1}^{\prime}=D_{5 \times 5} \bullet Q^{T} & =\left[\begin{array}{lllll}
0.05 & 0.1 & 0.2 & 0.3 & 0.2 \\
0.14 & 0.25 & 0.3 & 0.15 & 0.25 \\
0.2 & 0.25 & 0.35 & 0.15 & 0.05 \\
0.15 & 0.2 & 0.2 & 0.15 & 0.3 \\
0.3 & 0.2 & 0.15 & 0.15 & 0.1
\end{array}\right] \bullet(5,4,3,2,1)^{T}=\left[\begin{array}{l}
2.95 \\
2.8 \\
3.4 \\
3.34 \\
3.45
\end{array}\right] \\
C_{5 \times 1}^{\prime}=C_{5 \times 5} \bullet Q^{T} & =\left[\begin{array}{lllll}
0 & 0.2 & 0.25 & 0.3 & 0.2 \\
0.2 & 0.2 & 0.15 & 0.25 & 0.2 \\
0.15 & 0.2 & 0.2 & 0.25 & 0.15 \\
0.3 & 0.25 & 0.25 & 0.1 & 0.1 \\
0.345 & 0.2 & 0.1 & 0.3 & 0.15
\end{array}\right] \bullet(5,4,3,2,1)^{T}=\left[\begin{array}{l}
2.2 \\
2.85 \\
3.25 \\
3.45 \\
3
\end{array}\right] \\
E_{5 \times 1}^{\prime}=E_{3 \times 5} \bullet Q^{T} & =\left[\begin{array}{lllll}
0 & 0.1 & 0.2 & 0.2 & 0.5 \\
0.1 & 0.2 & 0.25 & 0.25 & 0.35 \\
0 & 0.1 & 0.3 & 0.2 & 0.25
\end{array}\right] \bullet(5,4,3,2,1)^{T}=\left[\begin{array}{l}
1.9 \\
2675 \\
2.15
\end{array}\right]
\end{aligned}
$$

(3) Calculation of membership value

The membership value of impact factors influencing the comprehensive evaluation results of the cloud safe storage evaluation model can be obtained according to the above membership functions obtained:

$$
D^{*}=f\left(S^{\prime}\right)=\left[\begin{array}{l}
0.795219958 \\
0.783970351 \\
0.849023523 \\
0.843207442 \\
0.854725151
\end{array}\right]
$$




$$
\begin{array}{r}
C^{*}=f\left(C^{\prime}\right)=\left[\begin{array}{l}
0.606511517 \\
0.792195455 \\
0.831343428 \\
0.865909854 \\
0.823584462
\end{array}\right] \\
E^{*}=f\left(E^{\prime}\right)=\left[\begin{array}{l}
0.477135618 \\
1.757498485 \\
0.623798474
\end{array}\right]
\end{array}
$$

\subsection{Calculation of storage model's performance and model evaluation value:}

As for the safety, convenience, economic of cloud safety storage, the relation setting weight can receive the weight vector $P=\left(P_{1}, P_{2}, P_{3}\right)$. Considering that the safety of the storage model is the most important, we should take care both safety, economic and convenience. Set the cloud safety storage model weight index as $P_{1}=50 \%$, $P_{2}=30 \%, P_{3}=20 \%$, the weight value of various influencing indicators in the cloud safe storage model should be set based on the corresponding influencing factors, for convenience, this paper adopts the average method for a calculation:

$$
\begin{gathered}
P_{1}=(0.3,0.3,0.3,0.3,0.3) \\
P_{2}=(0.2,0.2,0.2,0.2,0.2) \\
P_{3}=\left(\frac{1}{3}, \frac{1}{3}, \frac{1}{3}\right)
\end{gathered}
$$

Following the above steps, a weighted analysis of the influencing factors in performance indicators can give performance evaluation results of the cloud safe storage model:

$$
\begin{gathered}
D_{1}=P_{1} \bullet S^{*}=0.85622898 \\
D_{2}=P_{2} \bullet C^{*}=0.78907264 \\
D_{3}=P_{3} \bullet E^{*}=0.6186359348
\end{gathered}
$$

The comprehensive evaluation result can be obtained based on the evaluation result of each influencing factor of the cloud safe storage and the weight vector:

$$
\text { Score }=\left(D_{1}, D_{2}, D_{3}\right) \bullet\left(P_{1}, P_{2}, P_{3}\right)^{T}=0.799866553
$$

\subsection{Comprehensive performance evaluation of the cloud safe storage model}

The indicator evaluation value and the comprehensive evaluation value of each influencing factor can be obtained based on the previous calculations. This assay displays the results of AES encryption cloud safety storage model, DES encryption cloud safety storage model and encryption-based safety storage model, according to the evaluation process. 


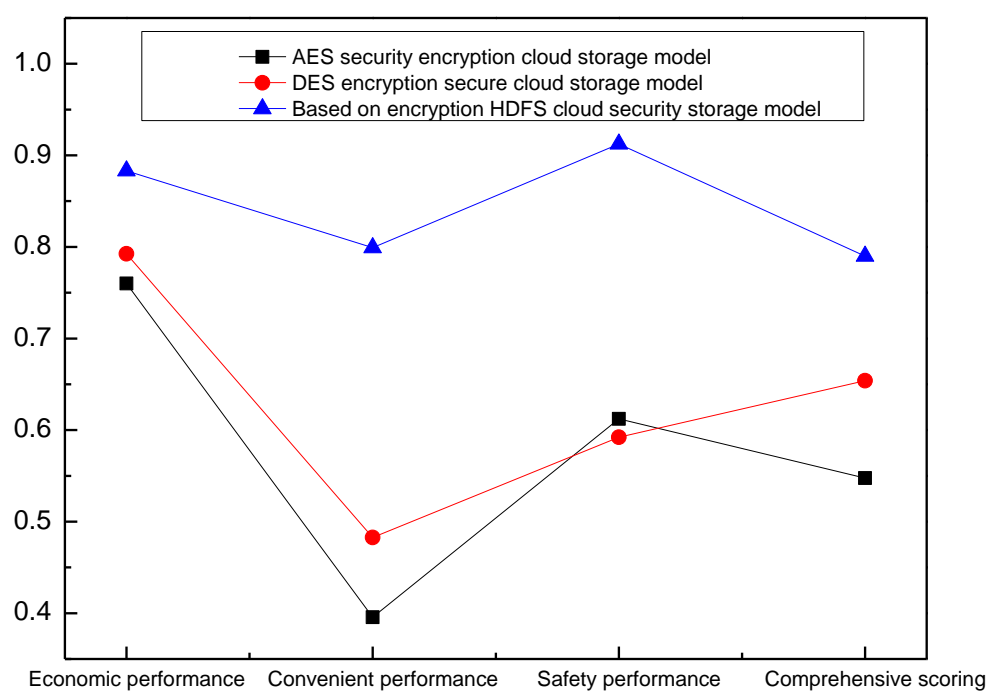

\section{Figure 1. The Analysis Results of Influencing Factors in Term of the Three Cloud Safe Storage Models}

Seen from the above picture, AES encryption cloud safety storage mudslides encryption cloud safety storage model and encryption-based safety storage model are in great advantage. Owing to the application of service tip encryption in cloud safety storage model, the safety of data is endured from resource.

In cloud safety storage model, encryption-based HDFS cloud storage model features in its convenience. Both sharing mechanism and secret paper have provided more convenient equipment for users 'using cloud data. So its comprehensive grade in convenience is far higher than other cloud safety storage model.

But in economic performance, encryption-based HDFS cloud safety storage model exist some shortcomings however. But the difference is not great comparing to other models.

\section{Conclusion}

This assay has analyzed the encryption-based HDFS safety storage model,DES encryption cloud safety storage model and AES encryption cloud safety storage model by designing the cloud storage evaluation model from the prospect of Internet finance. There we get following results:

(1) This paper makes analysis of economy, convenience and security of the cloud safe storage model and considers the statistical average the raw data, further improving accuracy of the cloud safe storage model evaluation.

(2) In the comprehensive evaluation of the cloud safe storage model, this paper provides valuable selective advice for the clients with different preferences based on dynamic performance index as the weight.

(3) The five-level evaluation mechanism is introduced in the cloud safe storage model to build evaluation system and membership functions are used to achieve the continuity of data-measuring, further proving a unifying evaluation mechanism for the cloud safe storage model.

The encrypt-based HDFS of cloud storage model should improve its calculator, lower the cost, and enhance the evaluation model mechanisms as to improve the suitability and correction. 


\section{References}

[1] Subramanian V, Seker R, Ramaswamy S, et al. PCIEF: a policy conflict identification and evaluation framework [J]. International Journal of Information and Computer Security, 2012, 5(1): 48-67.

[2] Gentry C. Fully homomorphism encryption using ideal lattices[C]//Proc of the 41st Annual ACM Symposium on Theory of Computing. New York: ACM Press, 2009:169-178

[3] Mansor A A, Wan Kadir W M N, Elias H,et al. Policy Overlap Analysis to Avoid Policy Conflict in Policy-based Management Systems[J]. International Journal of Innovative Computing, 2014, 4(1): 41-46.

[4] Gentry. C, Halevi. S. Fully homomorphism encryption without squashing using depth-3 arithmetic circuits[C]//Foundations of Computer Science (FOCS), 2011 IEEE 52nd Annual Symposium on IEEE, 2011:107-109

[5] Singh A N, Picot A, Kranz J, et al. Information Security Management (ISM) Practices: Lessons from Select Cases from India and Germany [J]. Global Journal of Flexible Systems Management, 2013, 14(4): 225-239.

[6] Van. Dijk. M, Gentry. C, Halevi.S,et. Fully homomorphic encryption over the integers [M] //Advances in Cryptology-EUROCRYPT 2010. Springer Berlin Heidelberg, 2010: 24-43

[7] Frenzel C, Sanneck H, Bauer B. A Fuzzy, Utility-Based Approach for Proactive Policy-Based Management[C]//Theoiy, Practice, and Applications of Rules on the Web. Springer Berlin Heidelberg, 2013: 84-98

[8] Coron. J. S, Mandal. A, Naccache.D. Fully Homomorphic encryption over the integers with shorter public keys [M]//Advances in Cryptology-CRYPTO 2011. Springer Berlin Heidelberg, 2011: 487-504

[9] Mell P, Grance T. The NIST Definition of Cloud Computing (draft) [M]. NIST Special Publication. 2011.

[10] Kim. J, Lee. M. S, Yun. A. CRT-based fully Homomorphic Encryption over the Integers [J]. IACR Cryptology Print Archive, 2013, 57.

[11] Klaudiusz Szczepaniak, Piotr Listos, Wojciech Lopuszynski et al. Granulomatous Peritonitis in a European Brown Bear Caused by Baylisascaris transfuga[J]. Journal of Wildlife Diseases, 2012, 48(2).

[12] Lei F, Shen-jun Y, Ting L et al. A Cloud Computing Application in Land Resources Information Management[C]: IEEE, 2010: 388-393

[13] W. Liu. Research on cloud computing security problem and strategy. in 2012 2nd International Conference on Consumer Electronics, Communications and Networks (CECNet), 2012, pp:1216-1219.

[14] M. Hamdi, "Security of cloud computing, storage, and networking," in 2012 International Conference on Collaboration Technologies and Systems (CTS), 2012, pp. 1-5.

[15] http://news.ccidnet.com/art/1032/20120504/3827437_1.html. An analysis of safety risk under cloud computering environment.

[16] Tim Mather, Subra Kumaraswamy, Shahed Latif. Cloud Security and Privacy [M]. Beijing: China Machine Press. 2011.

\section{Author}

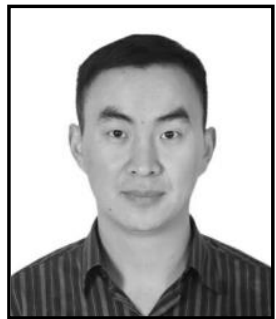

Hu yanqiang. He is a lecturer of Postdoctoral research center of Management Science and Engineering, School of management, Fudan University. His main research field is Financial management. 